\title{
Parâmetros biológicos e tabela de vida de fertilidade de Neopamera bilobata (Hemiptera: Rhyparochromidae) em morangueiro
}

\author{
Taciana Melissa de Azevedo Kuhn(1), Alci Enimar Loeck(1), Maria Aparecida Cassilha Zawadneak(2), \\ Mauro Silveira Garcia $^{(1)}$ e Marcos Botton ${ }^{(3)}$
}

(1)Universidade Federal de Pelotas, Faculdade de Agronomia Eliseu Maciel, Departamento de Fitossanidade, Caixa Postal 354, CEP 96010-970 Pelotas, RS, Brasil. E-mail: tacianakuhn@hotmail.com, alcienimar@yahoo.com.br, garciasmauro@yahoo.com.br (2)Universidade Federal do Paraná, Setor de Ciências Biológicas, Departamento de Patologia Básica, Caixa Postal 19.031, CEP 81531-990 Curitiba, PR, Brasil. E-mail: mazawa@ufpr.br ${ }^{(3)}$ Embrapa Uva e Vinho, Rua Livramento 515, Caixa Postal 130, CEP 95700-000 Bento Gonçalves, RS, Brasil. E-mail: marcos.botton@embrapa.br

Resumo - O objetivo deste trabalho foi avaliar os parâmetros biológicos e determinar a tabela de vida de fertilidade de Neopamera bilobata (Hemiptera: Rhyparochromidae) em morangueiro. O experimento foi realizado no Laboratório de Entomologia da Embrapa Uva e Vinho, em Bento Gonçalves, RS, em câmara climatizada (a $23 \pm 1{ }^{\circ} \mathrm{C}, 70 \pm 10 \%$ UR e fotófase de 12 horas). Utilizaram-se folíolos, flores, frutos verdes e maduros de morangueiro 'Aromas' como alimento para os percevejos. O desenvolvimento das fases imaturas e os parâmetros biológicos dos adultos foram monitorados diariamente. O percevejo $N$. bilobata não completou o ciclo biológico em folíolos e flores de morangueiro. Em frutos maduros e verdes, o tempo de desenvolvimento da ninfa ao adulto foi de $32,8 \pm 9,12$ e $36,7 \pm 6,80$ dias, com viabilidade de 27,3 e $51 \%$, respectivamente. O período de oviposição foi de 34,7 $\pm 21,2$ dias, com 319,1 $\pm 262,7$ ovos em frutos maduros, e de 43,9 $\pm 18,3$ dias, com 318,2 $\pm 144,7$ ovos em frutos verdes. Neopamera bilobata completa seu ciclo biológico na cultura do morangueiro. Frutos verdes de morangueiro são mais adequados ao desenvolvimento deste inseto do que frutos maduros.

Termos para indexação: Neopamera bilobata, Fragaria x ananassa, "catfacing", deformação dos frutos, pequenas frutas, percevejo.

\section{Biological parameters and fertility life table of Neopamera bilobata (Hemiptera: Rhyparochromidae) on strawberry}

\begin{abstract}
The objective of this work was to evaluate the biological parameters and to determinate the fertility life table of Neopamera bilobata (Hemiptera: Rhyparochromidae) on strawberry. The experiment was carried out in the Laboratory of Entomology of Embrapa Uva e Vinho, in Bento Gonçalves, RS, Brazil, in a climatic chamber (at $23 \pm 1{ }^{\circ} \mathrm{C}, 70 \pm 10 \% \mathrm{RH}$, and 12-hour photoperiod). Leaflets, flowers, and green and ripe fruit of 'Aromas' strawberry were used to feed the insects. The development of immature stages and the biological parameters of adults were daily monitored. The bug $N$. bilobata did not complete the life cycle on strawberry leaflets and flowers. On mature and green fruit, the nymph-adult development time was of $32.8 \pm 9.12$ and $36.7 \pm 6.80$ days, with viability of 27.3 and $51 \%$, respectively. The oviposition period was of $34.7 \pm 21.2$ days, with $319.1 \pm 262.7$ eggs on ripe fruit, and of $43.9 \pm 18.3$ days, with $318.2 \pm 144.7$ eggs on green fruit. Neopamera bilobata completes its biological cycle on strawberry crop. Green fruit of strawberry are more suitable for this insect development than ripe ones.
\end{abstract}

Index terms: Neopamera bilobata, Fragaria x ananassa, catfacing, fruit deformation, small fruit, bug.

\section{Introdução}

Entre as espécies de pequenas frutas, o morangueiro (Fragaria x ananassa) é a de maior área cultivada e a que está mais bem adaptada no Brasil. O cultivo está concentrado nos estados de Minas Gerais (41,4\%), Rio Grande do Sul (25,6\%), São Paulo (15,4\%), Paraná $(4,7 \%)$ e Distrito Federal (4\%), em uma área total de 3.500 hectares, com o cultivo praticado, principalmente, em pequenas propriedades (Pequenas frutas, 2005;
Antunes \& Reisser Junior, 2007). O interesse pela cultura é justificado em razão de sua alta rentabilidade, de ser uma fruta muito conhecida pelo consumidor e da diversidade de opções de comercialização e processamento (Fachinello et al., 2011)

A cultura é atacada por diversas espécies de insetos e ácaros (Botton \& Nava, 2010), e há relatos de Heteroptera alimentando-se dos frutos (GonzalesBustamante \& Díaz-Arriola, 1994; Easterbrook, 2000; Sweet, 2000). Nos Estados Unidos, Canadá e em países 
da Europa, os percevejos do gênero Lygus Hahn são os mais importantes, pois causam o dano conhecido como “catfacing”, uma deformação dos frutos decorrente da alimentação dos insetos nos aquênios (Allen \& Gaede, 1963; Jay et al., 2004; Labanowska, 2007).

No Brasil, insetos do gênero Lygus não foram relatados na cultura do morangueiro; porém, a ocorrência de altos índices de deformação dos frutos é frequente, com causas ainda pouco conhecidas. Recentemente, o percevejo identificado como Neopamera bilobata (Say, 1832) foi observado em altas populações na região Sul do Brasil. A espécie tem origem nos países da América do Norte (Integrated Taxonomic Information System, 2014), mas também há registros de ocorrência na América Central e do Sul (Melo et al., 2004). Os danos ocasionados pelo percevejo na cultura do morangueiro são: a paralização do crescimento, enrijecimento, secamento e coloração marrom dos pseudofrutos, em estágios iniciais de infestação. Em caso de elevada infestação, a coroa da planta também é atacada, o que causa murchamento (Brooks et al., 1929). A espécie é polífaga e pode alimentar-se também de plantas forrageiras (Cuda et al., 2007), florestais (Wheeler Junior \& Stoops, 2010), daninhas e frutíferas (Peredo \& Gamez-Virues, 2005).

Apesar da importância da espécie como causadora de deformações nos frutos, não foram encontrados dados de biologia de $N$. bilobata associada ao morangueiro, nem sobre as partes da planta em que o inseto se desenvolve. Essas informações permitiriam definir de forma apropriada as estratégias de monitoramento e controle.

O objetivo deste trabalho foi avaliar os parâmetros biológicos e determinar a tabela de vida de fertilidade de Neopamera bilobata (Hemiptera: Rhyparochromidae) em morangueiro.

\section{Material e Métodos}

O experimento foi realizado no Laboratório de Entomologia da Embrapa Uva e Vinho, em Bento Gonçalves, RS, com uso de câmara climatizada (BOD) a $23 \pm 1^{\circ} \mathrm{C}$, umidade relativa de $70 \pm 10 \%$ e fotófase de 12 horas.

Os exemplares utilizados para iniciar a criação em laboratório foram coletados de cultivos de morangueiro 'Albion', em Caxias do Sul, RS (29¹1'49"S e $\left.50^{\circ} 57^{\prime} 10^{\prime \prime} \mathrm{W}\right)$, e de morangueiro 'Aromas', em
Farroupilha, RS $\left(29^{\circ} 08^{\prime} 15^{\prime \prime} \mathrm{S}\right.$ e $\left.51^{\circ} 24^{\prime} 30^{\prime \prime} \mathrm{W}\right)$. A coleta foi feita pelo método de batida de plantas com vasilha de plástico branco (Van Driesche et al., 1998), e também manualmente, com recipientes de plástico cilíndricos com tampa de $80 \mathrm{~mL}$ (Ø $5 \mathrm{~cm}$ x $6 \mathrm{~cm}$ de altura).

Os percevejos foram enviados ao Dr. Pablo Matías Dellapé (Universidad Nacional de La Plata), que identificou a espécie-alvo, observada na cultura do morangueiro, como N. bilobata. "Voucher specimens" foram depositados no Museu Padre Jesus Santiago Moure (Universidade Federal do Paraná, Curitiba, PR) e na Coleção Entomológica da Embrapa Uva e Vinho.

Para a criação de adultos, a criação de manutenção foi estabelecida a $25 \pm 1^{\circ} \mathrm{C}$, com fotófase de 12 horas, em recipientes de plástico transparentes de $1 \mathrm{~L}$ (Ø 12 $\mathrm{cm} \times 9,5 \mathrm{~cm}$ de altura), com tampa perfurada $(4 \times 4 \mathrm{~cm})$ fechada com tecido tipo voil. Para as ninfas, a tampa foi substituída por um filme plástico. $\mathrm{O}$ fundo do recipiente foi forrado com papel-toalha, para evitar excesso de umidade. Como alimento, foram ofertados dois frutos maduros de morango por recipiente, trocados duas vezes por semana.

Foram utilizadas 150 ninfas com 24 horas de idade, para cada estrutura vegetal avaliada. As ninfas provieram de ovos depositados em placas de Petri, com papel absorvente (umedecido somente no primeiro dia). Os ovos - obtidos de posturas realizadas em papel-toalha e nas sépalas dos frutos de morango - foram coletados da criação de manutenção, com o auxílio de um pincel fino umedecido.

Como substrato de alimentação das ninfas, foram avaliados frutos verdes, maduros - com mais de $50 \%$ de coloração vermelha -, folíolos destacados de folhas completamente expandidas e flores. As partes da planta utilizadas como substrato de alimentação foram obtidas de cultivo estabelecido em casa de vegetação.

Folíolos e flores receberam, no local de secção, uma tira de algodão umedecido, coberto por papel absorvente, para evitar o contato direto dos insetos com o algodão e para manter a turgidez da estrutura vegetal. A troca destas estruturas foi realizada a cada dois dias, e a dos frutos maduros e verdes, duas vezes por semana.

Para o estudo da biologia, os insetos foram colocados em recipientes de plástico cilíndricos, com capacidade de $80 \mathrm{~mL}(\varnothing=5 \mathrm{~cm}$ e $6 \mathrm{~cm}$ de altura), com duas perfurações na tampa (cada uma com $1,5 \mathrm{~cm}$ de 
diâmetro), fechada com tecido voil aderido com cola vinil. Diariamente, avaliou-se o desenvolvimento do inseto, por meio do registro do momento da troca de instar. Após a emergência, os adultos foram separados por sexo, conforme Rodríguez S. (1998), e formaramse casais com fêmeas de no máximo 24 horas de idade e machos com até 48 horas, para cada substrato avaliado.

A avaliação diária da oviposição foi realizada pela contagem e retirada dos ovos, que foram acondicionados em tubos de microcentrífuga $(1,5 \mathrm{~mL})$, com tampa perfurada com alfinete entomológico número 01, para possibilitar a ventilação. As posturas foram avaliadas para se determinar a última postura viável; porém, para os cálculos da fase de ovo, utilizou-se a duração e viabilidade do segundo dia. Determinaram-se, para todo o período de desenvolvimento (ninfa-adulto), as viabilidades parcial e total e a razão sexual (número de fêmeas obtidas dividido pela soma de fêmeas e machos).

A comparação entre os dados de duração das fases imaturas e os demais parâmetros biológicos obtidos entre os diferentes substratos de alimentação foi feita quanto à normalidade, com o teste de Lilliefors. Posteriormente, as médias foram comparadas por meio do teste não paramétrico de Mann-Whitney, quando a distribuição da variável não apresentou normalidade, e pelo teste t de Student, para dados com distribuição normal, tendo-se utilizado o programa Assistat 7.7 beta.

A partir dos dados de longevidade e oviposição média, elaborou-se a tabela de vida e de fertilidade de $N$. bilobata, pelo cálculo do número médio de fêmeas produzidas por fêmea em cada data de oviposição $(\mathrm{mx})$ e em cada intervalo de tempo médio (x), tendose considerado o total de fêmeas vivas e o índice de sobrevivência acumulado de fêmeas (lx) durante o período de oviposição. Com base nas informações obtidas, estimaram-se os valores de IMG (intervalo médio entre gerações), $\mathrm{R}_{0}$ (taxa líquida de reprodução), rm (taxa intrínseca de crescimento) e $\lambda$ (razão finita de crescimento). $\mathrm{O}$ cálculo dos parâmetros biológicos e a comparação entre os tratamentos foram realizados pelo método Jackknife, com o programa SAS (Maia \& Luiz, 2006).

\section{Resultados e Discussão}

Observou-se que quando $N$. bilobata alimentou-se em folíolos e flores, os insetos não se desenvolveram; porém, houve sobrevivência das ninfas por $6,70 \pm 3,47$ dias (intervalo de 3-22 dias), nos insetos alimentados com flores, e por 4,12 $\pm 1,48$ dias (1-12 dias), nos alimentados com folíolos. Isso é uma indicação de que esses dois substratos não são adequados para o desenvolvimento do inseto, e podem ser usados apenas por breves períodos de tempo, quando não houver disponibilidade de frutos.

Fêmeas e machos de N. bilobata apresentaram cinco instares (Figura 1), conforme descrito por Peredo \& Gamez-Virues (2005) para espécimes de N. bilobata alimentadas com folhas e frutos de figo (Ficus spp. Linn.).

O tempo médio para o desenvolvimento da fase de ovo não diferiu significativamente quando os substratos foram fruto maduro ou verde (Tabela 1), com valores de 10,2 $\pm 0,69$ e 9,9 $\pm 0,73$ dias, respectivamente. O valor encontrado para a fase situou-se próximo ao relatado por Rodríguez S. (1998), que utilizou frutos de figo e sementes de girassol, e obteve uma duração entre 10 e 12 dias para N. bilobata. Peredo \& Gamez-Virues (2005) observaram duração de sete dias para insetos alimentados com frutos e folhas de figo $\left(20^{\circ} \mathrm{C}\right.$ e $70 \%$ UR).

A duração média do primeiro instar foi menor para insetos alimentados com frutos verdes $(8,2 \pm 2,67$ dias $)$ do que com frutos maduros (10,2 $\pm 3,36$ dias). Não houve diferença significativa entre as médias, quanto à duração do segundo $(7,0 \pm 3,23$ e $8,1 \pm 3,95$ dias $)$ e terceiro $(6,8 \pm 3,58$ e 7,9 $\pm 5,10$ dias) instares, para insetos alimentados com frutos verdes e maduros, respectivamente. No quarto e no quinto instares, o substrato fruto verde proporcionou maior duração das fases $(7,1 \pm 2,67$ e $8,4 \pm 1,89$ dias) do que o fruto maduro $(6,7 \pm 3,79$ e $6,6 \pm 1,99$ dias $)$.

A duração do período de desenvolvimento foi maior para insetos que se alimentaram do fruto verde (36,7 $\pm 6,80$ dias) do que com fruto maduro $(32,8 \pm 9,12$ dias); porém, a viabilidade total dos insetos em fruto verde $(51 \%)$ foi maior do que a observada nos frutos maduros $(27,3 \%)$.

Peredo \& Gamez-Virues (2005) observaram duração do ciclo biológico (ovo-adulto) em torno de 36 dias, em insetos alimentados em planta de figo, com período embrionário de sete dias e duração dos instares de $8,6,5,5$ e 5 dias, respectivamente para o primeiro, segundo, terceiro, quarto e quinto instares. Embora inferior, o valor encontrado por esses autores foi próximo ao obtido neste trabalho com morangueiro, o 
que indica que a cultura é um substrato adequado para o desenvolvimento do inseto.

Os valores de razão sexual foram de 0,47 e 0,51 , para insetos que emergiram de frutos maduros e verdes, respectivamente. Os substratos de alimentação avaliados não afetaram a longevidade das fêmeas,

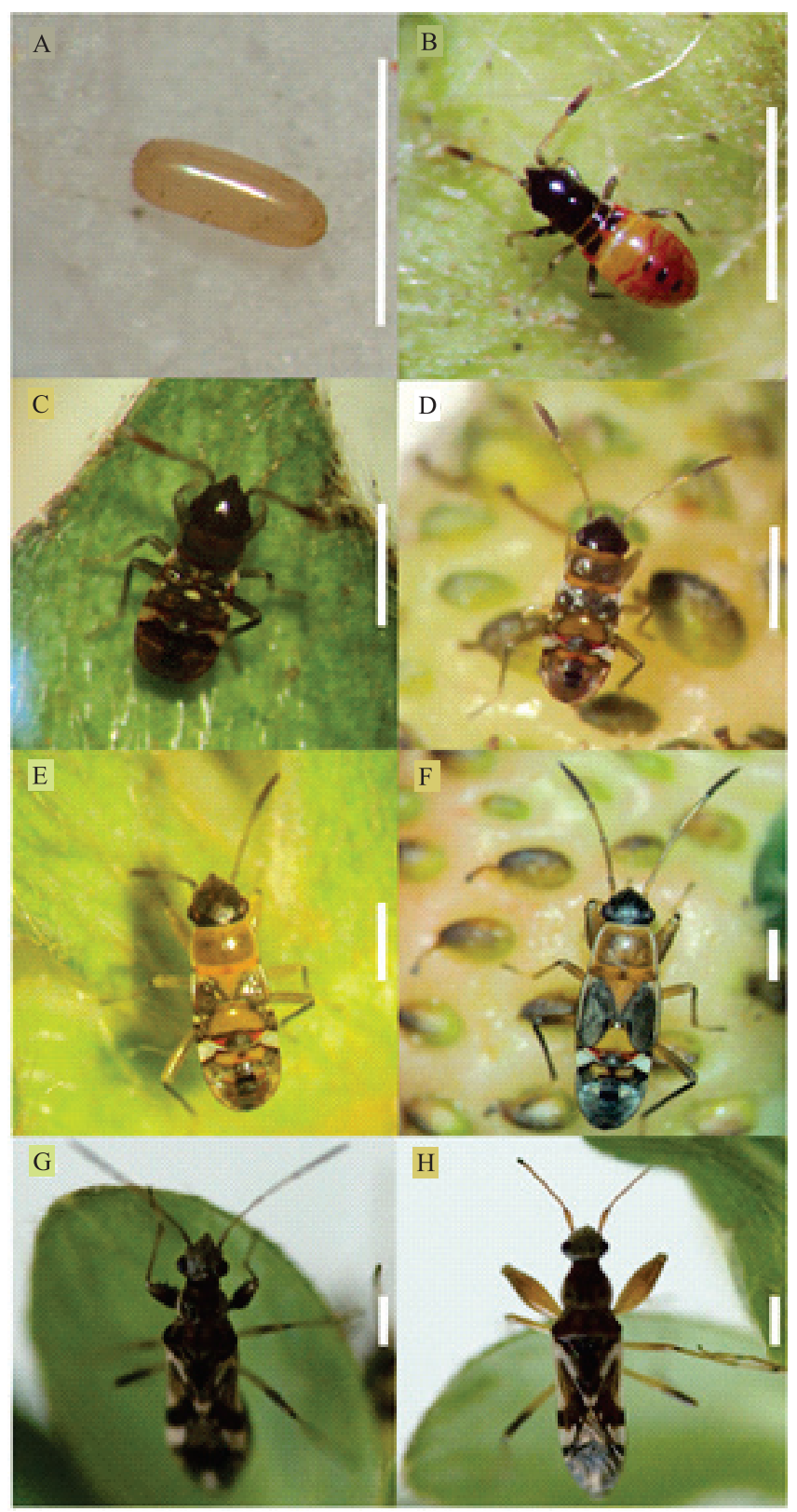

Figura 1. Fases de desenvolvimento de Neopamera bilobata: A, ovo; B, primeiro instar; C, segundo instar; D, terceiro instar; E, quarto instar; F, quinto instar; G, fase adulta de fêmea; e H, fase adulta de macho. Escala: $1 \mathrm{~mm}$. Fotos adultos: Vitor Cezar Pacheco da Silva. que foi de $40,6 \pm 24,9$ dias em frutos maduros e de $50,7 \pm 17,3$ dias em frutos verdes (Tabela 2). Embora a média observada tenha sido menor do que os 79 dias registrados por Rodríguez S. (1998), para $N$. bilobata, os valores do intervalo de variação foram maiores no presente trabalho, tendo chegado a 85 dias. A longevidade de machos alimentados não diferiu com frutos verdes $(71,6 \pm 33,01$ dias $)$ ou

Tabela 1. Duração média em dias, intervalo de variação (IV) e viabilidade (VB) em percentagem do primeiro, segundo, terceiro, quarto e quinto instares e do período de desenvolvimento de Neopamera bilobata alimentados com frutos maduro e verde de morangueiro cultivar Aromas $\left(23 \pm 1^{\circ} \mathrm{C}, 70 \pm 10 \% \text {, fotófase de } 12 \text { horas }\right)^{(1)}$.

\begin{tabular}{|c|c|c|c|c|c|c|c|c|}
\hline \multirow[t]{2}{*}{ Estádio } & \multicolumn{4}{|c|}{ Maduro } & \multicolumn{4}{|c|}{ Verde } \\
\hline & Duração & $\mathrm{n}$ & IV & VB & Duração & $\mathrm{n}$ & IV & VB \\
\hline $\mathrm{Ovo}^{(1)}$ & $10,2 \pm 0,69 a$ & 23 & $9-11$ & 27,7 & $9,9 \pm 0,73 \mathrm{a}$ & 152 & $8-13$ & 62,5 \\
\hline $1^{\circ}$ instar & $10,2 \pm 3,36 \mathrm{a}$ & 108 & $4-28$ & 85,2 & $8,2 \pm 2,67 b$ & 96 & $4-21$ & 73,9 \\
\hline $2^{\circ}$ instar & $8,1 \pm 3,95 \mathrm{a}$ & 86 & $2-19$ & 78,9 & $7,0 \pm 3,23 \mathrm{a}$ & 79 & $2-19$ & 82,3 \\
\hline $3^{\circ}$ instar & $7,9 \pm 5,10 \mathrm{a}$ & 73 & $2-23$ & 84,9 & $6,8 \pm 3,58 \mathrm{a}$ & 74 & $2-21$ & 93,7 \\
\hline $4^{\circ}$ instar & $6,7 \pm 3,79 b$ & 51 & $2-17$ & 69,9 & $7,1 \pm 2,67 \mathrm{a}$ & 70 & $3-15$ & 94,6 \\
\hline $5^{\circ}$ instar & $6,6 \pm 1,99 b$ & 35 & $4-12$ & 68,8 & $8,4 \pm 1,89 a$ & 65 & $3-13$ & 94,3 \\
\hline Ninfa/adul & $32,8 \pm 9,12 b$ & 35 & $21-57$ & 27,3 & $36,7 \pm 6,80 \mathrm{a}$ & 65 & $18-56$ & 51,0 \\
\hline
\end{tabular}

${ }^{(1)}$ Médias \pm desvio padrão seguidas por letras iguais, nas linhas, não diferem entre si, pelo teste U de Mann-Whitney, para os estádios ovo e ninfa, e pelo teste $\mathrm{t}$ de Student, para o período de desenvolvimento, a 5\% de probabilidade. n, número de observações.

Tabela 2. Média e intervalos de variação (IV) da longevidade de fêmeas e machos; dos períodos de pré-oviposição, oviposição e pós-oviposição (dias); e da fecundidade diária e total de Neopamera bilobata alimentada com frutos maduros e verdes de morangueiro 'Aromas' $\left(23 \pm 1^{\circ} \mathrm{C}\right.$; $70 \pm 10 \%$; fotófase de 12 horas) ${ }^{(1)}$.

\begin{tabular}{|c|c|c|c|c|c|c|}
\hline \multirow[t]{2}{*}{ Parâmetro } & \multicolumn{3}{|c|}{ Maduro } & \multicolumn{3}{|c|}{ Verde } \\
\hline & Média & $\mathrm{n}$ & IV & Média & $\mathrm{n}$ & IV \\
\hline & \multicolumn{6}{|c|}{ Longevidade } \\
\hline Fêmeas & $40,6 \pm 24,9 \mathrm{aB}$ & 8 & $11-85$ & $50,7 \pm 17,3 \mathrm{aB}$ & 22 & $25-85$ \\
\hline \multirow[t]{2}{*}{ Machos } & $86,3 \pm 31,8 \mathrm{aA}$ & 7 & $38-122$ & $71,6 \pm 33,01 \mathrm{aA}$ & 14 & $10-111$ \\
\hline & \multicolumn{6}{|c|}{ Período (dias) } \\
\hline Pré-oviposição & $4,3 \pm 1,50 \mathrm{a}$ & 9 & $2-7$ & $4,6 \pm 1,47 a$ & 23 & $3-9$ \\
\hline Oviposição & $34,7 \pm 21,2 \mathrm{a}$ & 9 & $2-63$ & $43,9 \pm 18,3 \mathrm{a}$ & 23 & $12-81$ \\
\hline Oviposição viável & $34,8 \pm 18,0 \mathrm{a}$ & 9 & $9-60$ & $41,1 \pm 13,6 \mathrm{a}$ & 23 & $21-77$ \\
\hline \multirow[t]{2}{*}{ Pós-oviposição } & $5,4 \pm 13,2 \mathrm{a}$ & 8 & $0-38$ & $4,0 \pm 4,7 \mathrm{~b}$ & 22 & $1-21$ \\
\hline & \multicolumn{6}{|c|}{ Fecundidade (ovos) } \\
\hline Diária & $8,6 \pm 3,74 a$ & 9 & $4-13,6$ & $8,2 \pm 2,45 \mathrm{a}$ & 23 & $2-11,9$ \\
\hline Total & $319,1 \pm 262,7 \mathrm{a}$ & 9 & $12-668$ & $318,2 \pm 144,7 \mathrm{a}$ & 23 & $11-564$ \\
\hline
\end{tabular}

${ }^{(1)}$ Médias \pm desvio padrão seguidas por letras iguais, minúscula na linha e maiúscula na coluna, não diferem entre si, pelo teste U de Mann-Whitney, para os parâmetros pré- e pós-oviposição, e pelo teste t de Student, para os demais parâmetros, a 5\% de probabilidade. n, número de observações. 
maduros (86,3 $\pm 31,8$ dias), e foi maior do que a das fêmeas. Peredo \& Gamez-Virues (2005) observaram longevidade de adultos de 22 dias, valor abaixo do registrado no presente estudo.

O período de pré-oviposição e de oviposição foram iguais, nos diferentes substratos (Tabela 2). O período de pós-oviposição, no entanto, foi maior em fêmeas alimentadas com frutos maduros $(5,4 \pm 13,2$ dias $)$ do que com frutos verdes $(4,0 \pm 4,7$ dias $)$.

A fecundidade diária obtida em frutos maduros $(8,6 \pm 3,74$ ovos $)$ não diferiu da obtida em frutos verdes $(8,2 \pm 2,45$ ovos), e a fecundidade total, nos frutos maduros $(319,1 \pm 262,7$ ovos) não diferiu da obtida nos frutos verdes $(318,7 \pm 144,7$ ovos). Esses valores foram superiores aos 2,2 ovos por dia e 155 ovos registrados por Rodríguez S. (1998) para N. bilobata. $\mathrm{O}$ autor observou apenas uma fêmea, alimentada em dias alternados; portanto, seus resultados podem estar subestimados, o que explicaria a diferença observada.

Fêmeas virgens realizaram oviposição de ovos inférteis. Não houve diferença significativa entre os substratos, quanto à viabilidade de ovos das fêmeas que realizaram cópula, do período de emergência da fêmea até o dia da última oviposição viável: $34,8 \pm 18,0$ dias, em frutos maduros, e de 41,1 $\pm 13,6$ dias em frutos verdes (Tabela 2). O tempo de oviposição de posturas viáveis foi maior do que o registrado por Rodríguez S. (1998) para $N$. bilobata, que relatou que ovos colocados após o $40^{\circ}$ dia de vida da fêmea foram inférteis.

No que se refere ao intervalo entre gerações (IMG) também não houve diferença entre frutos verdes (70,1 dias) e frutos maduros (67,4 dias) (Tabela 3).

Quanto à taxa líquida de reprodução $\left(\mathrm{R}_{0}\right)$, que é o número de vezes que a população aumenta a cada geração, o valor encontrado em frutos verdes $(50,5)$ foi mais de três vezes maior do que o registrado em frutos maduros $(13,9)$, em razão da baixa viabilidade das fases de ovo e ninfa no

Tabela 3. Intervalo entre gerações (IMG), taxa líquida de reprodução $\left(R_{0}\right)$, taxa intrínseca de crescimento $(\mathrm{rm})$ e taxa finita de aumento $(\lambda)$ de Neopamera bilobata alimentada com o frutos maduros e verdes de morangueiro 'Aromas' $\left(23 \pm 1^{\circ} \mathrm{C} ; 70 \pm 10 \% \text {; fotófase de } 12 \text { horas }\right)^{(1)}$.

\begin{tabular}{lcccc}
\hline Substrato & IMG (dias) & $\mathrm{R}_{0}$ & $\mathrm{rm}$ & $\lambda$ \\
\hline Fruto maduro & $67,4 \mathrm{a}$ & $13,9 \mathrm{~b}$ & $0,0390 \mathrm{~b}$ & $1,0398 \mathrm{~b}$ \\
Fruto verde & $70,1 \mathrm{a}$ & $50,5 \mathrm{a}$ & $0,0559 \mathrm{a}$ & $1,0575 \mathrm{a}$ \\
\hline
\end{tabular}

${ }^{(1)}$ Médias seguidas de letras iguais não diferem pelo método Jackknife, a $5 \%$ de probabilidade. segundo substrato. A capacidade inata de aumentar em número ( $\mathrm{rm})$ foi maior com frutos verdes $(0,0559)$ do que com maduros $(0,0390)$, o que indica maior velocidade de crescimento da população nos frutos em desenvolvimento. Quanto à taxa finita de aumento $(\lambda)$, o valor obtido indicou que o número de indivíduos adicionados à população a cada geração foi maior nos fruto verdes $(1,0575)$ do que nos maduros $(1,0398)$.

Os valores observados de $\mathrm{R}_{0}$, rm e $\lambda$ indicam que a população de $N$. bilobata apresenta melhor desempenho em frutos verdes do que apenas em frutos maduros. Como a planta de morangueiro apresenta os dois substratos disponíveis praticamente durante todo o período reprodutivo, os resultados observados indicam que a população do inseto é capaz de aumentar em número ao longo desse período, e que, dependendo da temperatura, podem ocorrer altas populações no pico da safra.

Além dos resultados encontrados, no presente trabalho foi observado o comportamento de canibalismo na espécie quando os frutos não apresentaram condições ideais, como nos casos em que estavam ressecados. Relatos de canibalismo também não são incomuns nos percevejos sugadores de sementes (Hemiptera: Rhyparochrominae) (Eyles, 1964). Além disso, Buschman et al. (1977) relataram que o percevejo $N$. bilobata são capazes de predar ovos de Anticarsia gemmatalis Hübner. Estas observações indicam que $N$. bilobata, assim como outros percevejos de sementes, pode complementar sua dieta vegetal com alimentação animal.

\section{Conclusões}

1. Neopamera bilobata completa seu ciclo biológico na cultura do morangueiro.

2. Frutos verdes de morangueiro são mais adequados ao desenvolvimento de $N$. bilobata do que frutos maduros.

3. Folhas e flores de morangueiro não são adequados ao desenvolvimento de $N$. bilobata.

\section{Agradecimentos}

À Coordenação de Aperfeiçoamento de Pessoal de Nível Superior (Capes), por concessão de bolsa; à Universidade Federal de Pelotas (UFPel), pelo auxílio financeiro; e à Embrapa Uva e Vinho(CNPUV), pelo auxílio com infraestrutura. 


\section{Referências}

ALLEN, W.W.; GAEDE, S.E. The relationship of Lygus bugs and thrips to fruit deformity in strawberries. Journal of Economic Entomology, v.56, p.823-825, 1963.

ANTUNES, L.E.C.; REISSER JUNIOR, C. Fragole, i produttori brasiliani mirano all'esportazione in Europa. Rivista di Frutticoltura e Ortofloricoltura, v.69, p.60-64, 2007.

AZEVEDO FILHO, W.S.; BOTTON, M.; SORIA, S. de J. Curadoria da coleção entomológica da Embrapa Uva e Vinho. Bento Gonçalves: Embrapa Uva e Vinho, 2007. (Embrapa Uva e Vinho. Comunicado técnico, 77).

BOTTON, M.; NAVA, D.E. Principais pragas do morangueiro. Revista Campo e Negócios, v.6, p.74-80, 2010.

BROOKS A.N.; WATSON, J.R.; MOWRY, H. Strawberries in Florida: culture, diseases and insects. Gainesville: University of Florida Agricultural Experiment Station, 1929. 523p.

BUSCHMAN, L.L.; WHITCOMB, W.H.; HEMENWAY, R.C.; MAYS, D.L.; NGUYEN, R.U.; LEPPLA, N.C.; SMITTLE, B.J. Predators of velvetbean caterpillar eggs in Florida soybeans. Environmental Entomology, v.6, p.403-407, 1977.

CUDA, J.P.; DUNFORD, J.C.; LEAVENGOOD JUNIOR, J.M. Invertebrate fauna associated with torpedograss, Panicum repens (Cyperales: Poaceae), in Lake Okeechobee, Florida, and prospects for biological control. Florida Entomologist, v.90, p.238-248, 2007. DOI: 10.1653/0015-4040(2007)90[238:IFAW $\mathrm{TP}] 2.0 . \mathrm{CO} ; 2$.

EASTERBROOK, M.A. Relationships between the occurrence of misshapen fruit on late-season strawberry in the United Kingdom and infestation by insects, particularly the European tarnished plant bug, Lygus rugulipennis. Entomologia Experimentalis et Applicata, v.96, p.59-67, 2000. DOI: 10.1046/j.15707458.2000.00679.x.

EYLES, A.C. Feeding habits of some Rhyparochrominae (Heteroptera: Lygaeidae) with particular reference to the value of natural foods. Transactions of the Royal Entomological Society, v.116, p.89-114, 1964. DOI: 10.1111/j.1365-2311.1964. tb00826.x.

FACHINELLO, J.C.; PASA, M. da S.; SCHMTIZ, J.D.; BETEMPS, D.L. Situação e perspectivas da fruticultura de clima temperado no Brasil. Revista Brasileira de Fruticultura, v.33, p.109-120, 2011. DOI: $10.1590 / \mathrm{S} 0100-29452011000500014$.

GONZALES-BUSTAMANTE, L.; DÍAZ-ARRIOLA, S. Nysius sp. (Hemiptera-Lygaeidae) en fresa cultivada en el Valle Huaral (Lima). Revista Peruana de Entomología, v.36, p.19-21, 1994.
INTEGRATED TAXONOMIC INFORMATION SYSTEM. Neopamera bilobata. Available at: <http:/www.itis.gov/servlet/ SingleRpt/SingleRpt?search_topic $=$ TSN\&search_value $=108084>$. Accessed on: 28 May 2014.

JAY, C.N.; CROSS, J.V.; BURGESS, C. The relationship between populations of European tarnished plant bug (Lygus rugulipennis) and crop losses due to fruit malformation in everbearer strawberries. Crop Protection, v.23, p.825-834, 2004. DOI: 10.1016/j. cropro.2004.01.002.

LABANOWSKA, B.H. Strawberry fruit damaged by the tarnished plant bug (Lygus rugulipennis L.). Journal of Fruit and Ornamental Plant Research, v.15, p.147-156, 2007.

MAIA, A.H.N.; LUIZ, A.J.B. Programa SAS para análise de tabelas de vida e fertilidade de artrópodes: o método jackknife. Jaguariúna: Embrapa Meio Ambiente, 2006. 11p. (Embrapa Meio Ambiente. Comunicado técnico, 33).

MELO, M.C.; DELLAPÉ, P.M.; CARPINTERO, D.L.; COSCARÓ, M. del C. Reduviidae, Miridae y Lygaeoidea (Hemiptera) recolectados en Colonia Carlos Pellegrini (Esteros de Iberá, Corrientes, Argentina). Revista de la Sociedad Entomológica Argentina, v.63, p.59-67, 2004.

PEQUENAS frutas. Anuário Brasileiro da Fruticultura, p.9097, 2005.

PEREDO, L.C.; GAMEZ-VIRUES, S. Three species of facultative Myodochini (Lygaeoidea: Rhyparochromidae) associated with figs in Mexico. Proceedings of the Entomological Society of Washington, v.107, p.362-375, 2005.

RODRÍGUEZ S., R.L. Copulation, fighting behavior and life cycle of Neopamera bilobata (Heteroptera: Lygaeidae). Revista de Biología Tropical, v.46, p.837-840, 1998.

SWEET, M.H. Seed and chinch bugs (Lygaeoidea). In: SCHAEFER. C.W.; PANIZZI, A.R. Heteroptera of economic importance. Boca Raton: CRC Press, 2000. p.143-262. DOI: 10.1201/9781420041859.ch6.

VAN DRIESCHE, R.G.; HEINZ, K.M.; VAN LENTEREN, J.C.; LOOMANS, A.; WICK, R.; SMITH, T.; LOPES, P.; SANDERSON, J.P.; DAUGHTREY, M.; BROWNBRIDGE, M. Western flower thrips in greenhouses: a review of its biological control and other methods. Amherst: University of Massachusetts Amherst, 1998. 31p.

WHEELER JUNIOR, A.G.; STOOPS, C.A. Cnemodus hirtipes Blatchley and C. mavortius (Say) (Hemiptera: Lygaeoidea: Rhyparochromidae) in fallen pine cones, with consideration of the biological significance of cone occupancy. Proceedings of the Entomological Society of Washington, v.112, p.155-168, 2010. DOI: $10.4289 / 0013-8797-112.1 .155$.

Recebido em 11 de fevereiro de 2014 e aprovado em 30 de maio de 2014 